\title{
Ultrastructural Analysis of Substance P-Immunoreactive Nerve Fibers in Myenteric Ganglia of Guinea Pig Small Intestine
}

\author{
I. J. Llewellyn-Smith, J. B. Furness, and M. Costa \\ Departments of Anatomy and Histology and of Physiology and Centre for Neuroscience, School of Medicine, Flinders \\ University, Bedford Park, South Australia 5042, Australia
}

Electron microscopic immunocytochemistry has been used to study the distribution and synaptic relationships of substance P (SP)-immunoreactive nerve fibers in ultrathin sections from whole-mount preparations of myenteric ganglia from guinea pig small intestine. At the light microscopic level, myenteric ganglia stained for ultrastructural study contained dense arrays of SP-immunoreactive nerve fibers around and between the neuronal and glial cell bodies. At the electron microscopic level, SP-containing nerve fiber profiles occurred throughout the neuropil of the myenteric ganglia. Both vesicle-containing and nonvesiculated nerve fiber profiles were immunoreactive. The positive vesiculated profiles contained variable proportions of small clear and large granular vesicles. Two-thirds of the vesicle-containing nerve fiber profiles in myenteric ganglia were immunoreactive for SP. Many vesiculated SP-immunoreactive nerve fiber profiles were directly apposed to each myenteric neuron. However, only about $0.6 \%$ of these vesiculated profiles formed synapses that showed the ultrastructural specializations of vesicle clustering presynaptically and fuzzy electron-dense material on the postsynaptic membrane. On the other hand, morphologically identifiable synapses with SP-immunoreactivity comprised about half the total number of synapses in the ganglia. SP-immunoreactive synapses were observed on nonvesiculated nerve processes in the periphery of the ganglla and on nerve cell bodies. Most of the axosomatic SP synapses occurred on small neurons that lay either on the surfaces of the ganglia or near the fibers of the internodal strands where they traveled through the ganglia. Both SP. positive and SP-negative nerve cell bodies received SP synapses. These results show that synapses in which the presynaptic element is SP-immunoreactive make up a high proportion of the total number of morphologically identifiable synapses in guinea pig myentric ganglia. They suggest that, although all myenteric neurons are directly contacted by SP-immunoreactive varicosities, a subpopulation of myenteric neurons preferentially receives SP synapses that show clear pre- and postsynaptic specializations.

\footnotetext{
Received Nov. 27, 1987; revised Apr. 28, 1988; accepted June 14, 1988.

This work was supported by a grant from the National Health and Medical Research Council of Australia. We wish to thank Dianne Trussell for expert technical assistance and Dr. Roger Murphy for the anti-substance P antiserum.

Correspondence should be addressed to Dr. I. J. Llewellyn-Smith, Department of Medicine, School of Medicine, Flinders University, Bedford Park, South Australia 5042, Australia.

Copyright (C) 1989 Society for Neuroscience $0270-6474 / 89 / 010167-08 \$ 02.00 / 0$
}

Since the observations of von Euler and Gaddum (1931) that substance $\mathrm{P}$ (SP) occurs in extracts of the intestine and is a powerful stimulant of intestinal smooth muscle, there have been many investigations into the possible roles that SP might play in the gut. SP stimulates the release of $\mathrm{ACh}$ from intestinal neurons, has direct contractile effects on intestinal smooth muscle, and may be involved in the nervous mechanisms underlying peristalysis (Hedqvist and von Euler, 1975; Holzer and Lembeck, 1979, 1980; Holzer et al., 1981; Tonini et al., 1981; Bartho et al., 1982; Holzer, 1984; reviewed by Pernow, 1983; Bartho and Holzer, 1985; Costa et al., 1986b). Very dense networks of SP-containing axons, as well as SP-immunoreactive nerve cell bodies, occur in myenteric and submucous ganglia in all species studied to date (reviewed by Furness et al., 1988b). Pharmacological and radioimmunoassay studies indicate that $\mathrm{SP}$ is released from intestinal neurons (Franco et al., 1979; Leander et al., 1981; Baron et al., 1983; Holzer 1984). SP has been shown to produce concentration-dependent depolarizations in most myenteric neurons (Katayama and North, 1978; Grafe et al., 1979; Katayama et al., 1979) and is likely to contribute to slow excitatory potentials (esps) in myenteric ganglia (Morita et al., 1980; Johnson et al., 1981; Bornstein et al., 1984b). SP-immunoreactive nerve fibers form synapses on nerve cell bodies and nerve processes in rat, cat, and human myenteric ganglia (Fehér and Wenger, 1981; Fehér et al., 1984; Llewellyn-Smith et al., 1984).

No detailed ultrastructural study has yet been published on SP-immunoreactive nerve fibers in myenteric ganglia from guinea pig small intestine, where most of the pharmacological and electrophysiological studies have been done. We undertook the present investigation to see whether we could gain some insight into the functions of SP fibers from their ultrastructural distribution and relations to other nerve cell bodies and fibers within the ganglia. We were also interested in determining whether SPimmunoreactive varicositics showed any ultrastructural peculiarities that might allow them to be recognized in conventionally fixed samples of intestine. We used a technique for electron microscopic immunocytochemistry on whole-mount preparations of myenteric ganglia (Llewellyn-Smith et al., 1985) to study these questions. Since this method allows both immunoreactive and nonimmunoreactive fibers to be observed and counted in whole ganglia, we have also been able to determine the proportion of SP-immunoreactive varicosities and the proportion of SP-positive synapses within the ganglia.

\section{Materials and Methods}

Electron microscopic immunocytochemistry. Eleven male or female guinea pigs weighing $300-500 \mathrm{gm}$ were used. Segments of intestine were pro- 

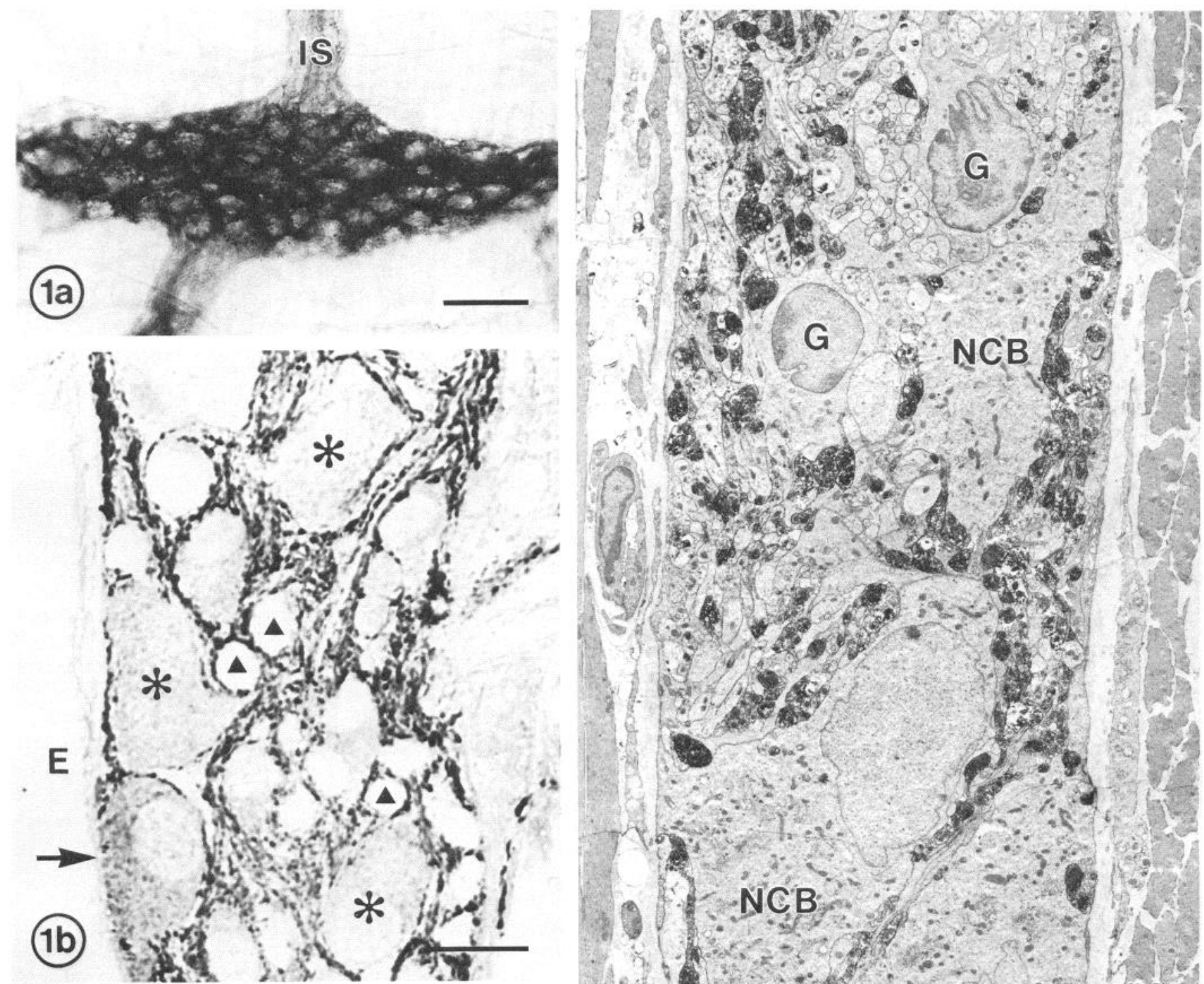

(1a)

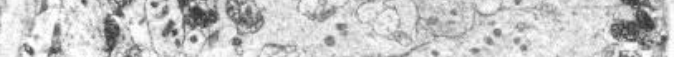

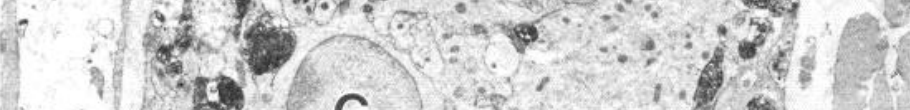
G G G. NCB a.

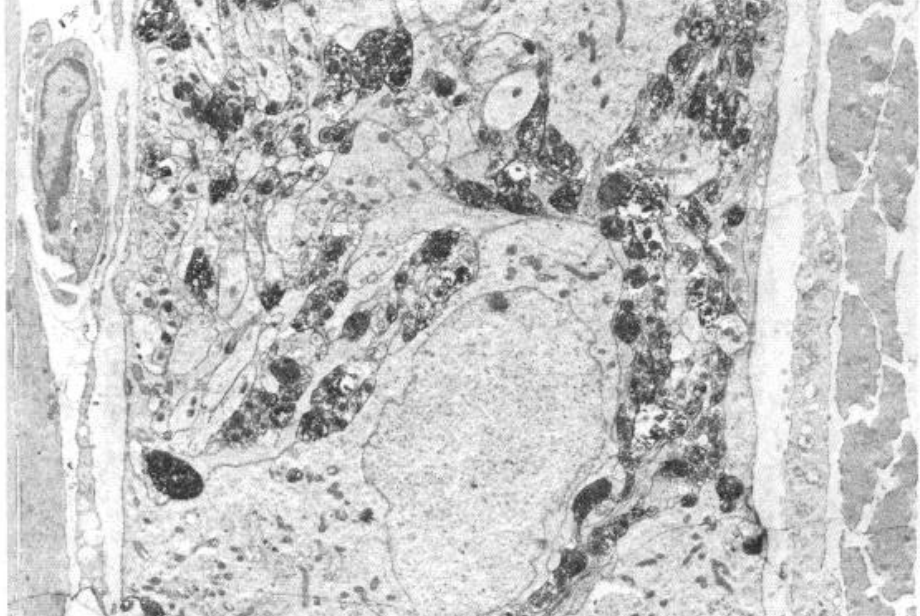

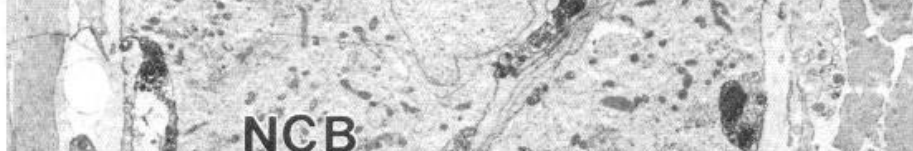
13 NCB $^{-1}=\ldots$

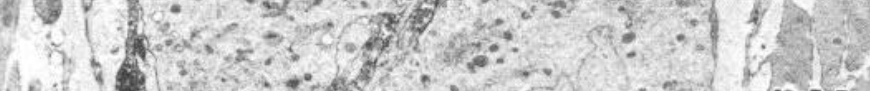

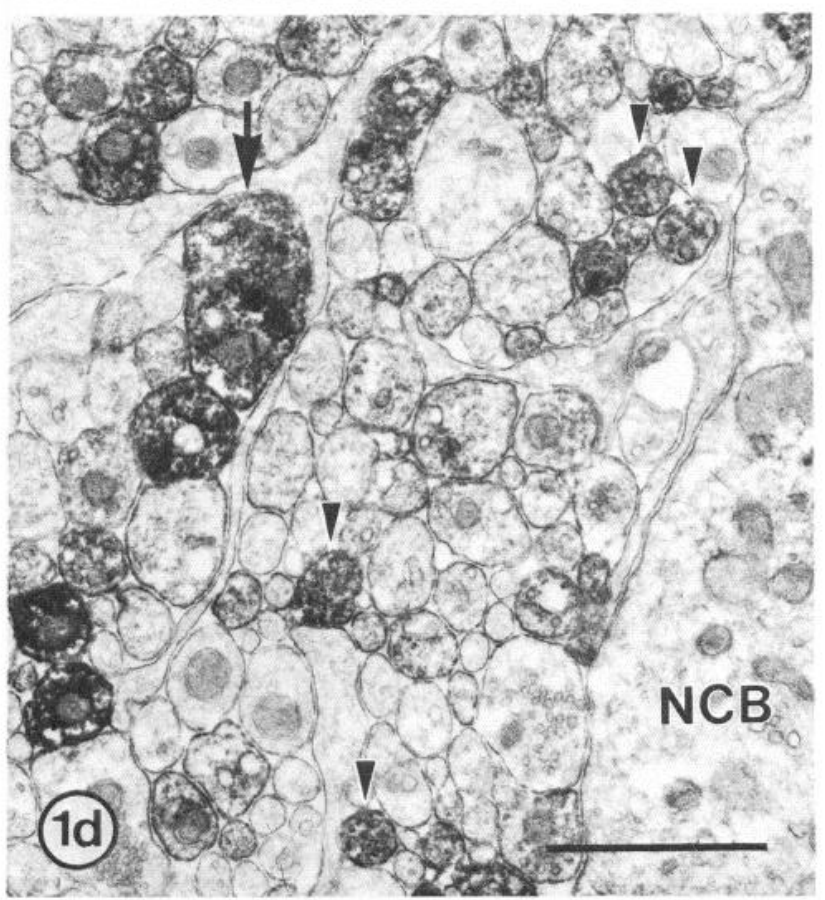

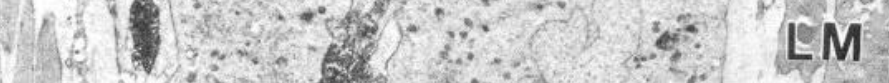

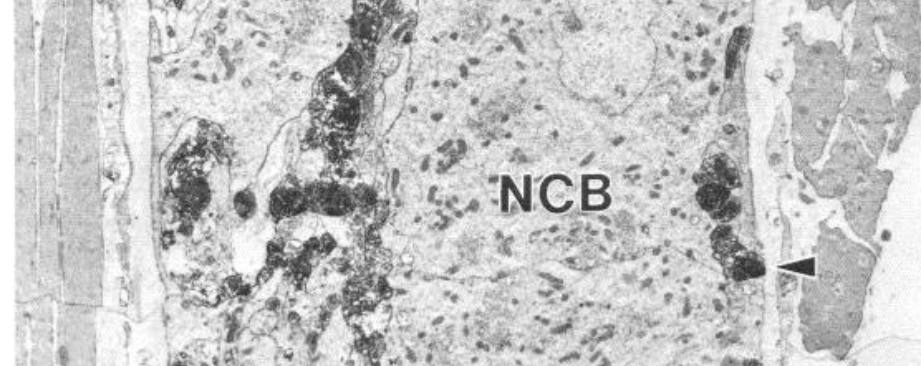

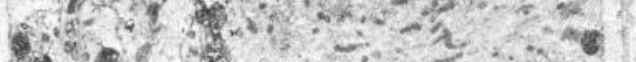

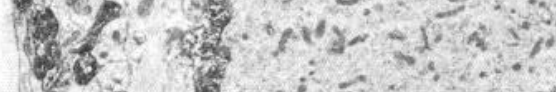

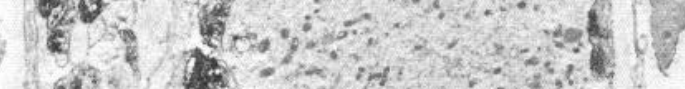

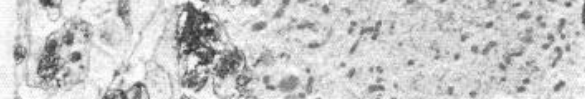

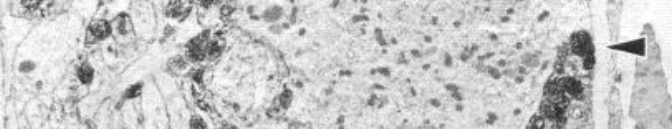
CM

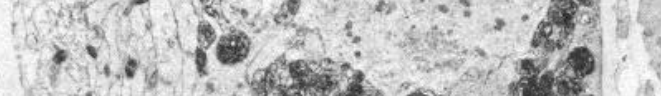

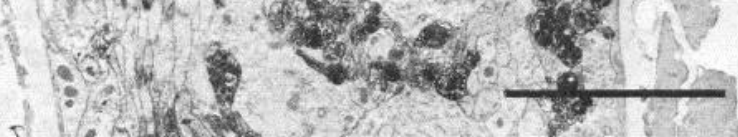


cessed for electron microscopic immunocytochemistry on whole-mount preparations using the method of Llewellyn-Smith et al. (1985) with minor modifications. Briefly, pieces of gut were taken from the distal third of the small intestine of all animals and from the proximal third of the intestine of one animal. The intestinal segment from one animal had been extrinsically denervated (see Llewellyn-Smith et al., 1988b). The gut segments were pinned on balsa wood, incubated for 1-2 hr in tissue culture medium (DME/F12 Ham; Sigma, St. Louis) that was bubbled with medical air containing $5 \% \mathrm{CO}_{2}$ and then fixed by immersion for $1 \mathrm{hr}$ in $4 \%$ formaldehyde, $0.05 \%$ glutaraldehyde, $0.2 \%$ picric acid in $0.1 \mathrm{M}$ phosphate buffer, $\mathrm{pH}$ 7.4. The fixed tissue was chopped into $1 \mathrm{~cm}^{2}$ pieces, washed vigorously in $3-4 \times 10 \mathrm{~min}$ changes of $50 \%$ ethanol, washed in several changes of phosphate buffer, and stored in buffer overnight at $4^{\circ} \mathrm{C}$. The tissue was then treated with $0.1 \% \mathrm{NaCNBH}_{3}$ in phosphate buffer for $30 \mathrm{~min}$ and washed $3 \times 15 \mathrm{~min}$ in phosphatebuffered saline (PBS). Whole mounts containing myenteric plexus and longitudinal muscle or myentric plexus and circular muscle were prepared.

The whole mounts were placed in $1 \%$ normal goat serum (NGS) in PBS containing $0.05 \%$ merthiolate for at least $30 \mathrm{~min}$ and then incubated for about $65 \mathrm{hr}$ in a 1:16,000 dilution of anti-SP antiserum (RMSP I/ 2; Morris et al., 1986). The whole mounts were transferred to a 1:200 dilution of biotinylated anti-rabbit IgG for $24 \mathrm{hr}$ and then to avidinbiotin-HRP (Vectastain ABC kit; Vector Laboratories, Burlingame, CA) for $6 \mathrm{hr}$. Both primary and secondary antibodies were made up in PBSmerthiolate and contained $1 \% \mathrm{NGS}$; the $\mathrm{ABC}$ reagent was prepared in PBS-merthiolate. All incubations were carried out at room temperature in a humid chamber. After each antibody incubation, the tissues were washed in $3 \times 10$ min changes of PBS with agitation.

To reveal the immunoreactive nerve fibers in the ganglia, the whole mounts were immersed for $10 \mathrm{~min}$ in a solution containing $0.5 \mathrm{mg} / \mathrm{ml}$ diaminobcnzidinc (DAB) in $50 \mathrm{~mm}$ Tris-HCl, $\mathrm{pH} 7.6$, with or without $10 \mathrm{~mm}$ imidazole to intensify the reaction (Straus, 1982). A peroxidase reaction was then carried out for $8 \mathrm{~min}$ in a fresh solution of the same composition to which $\mathrm{H}_{2} \mathrm{O}_{2}$ had been added to give a final concentration of $0.005 \%$.

For controls, whole mounts of myenteric plexus were incubated without primary antiserum or with anti-SP antiserum that had been absorbed with $10^{-4}, 10^{-5}$, or $10^{-6} \mathrm{M} \mathrm{SP}$ and then stained as above.

After several PBS washes and overnight storage, the antibody-labeled whole mounts were postfixed in $0.5 \% \mathrm{OsO}_{4}$ and stained en bloc with $2 \%$ aqueous uranyl acetate. The tissue was then dehydrated through alcohol and acetone, infiltrated, embedded in TAAB embedding resin under greasy coverslips on flat blank blocks, and polymerized for 24$48 \mathrm{hr}$. Ganglia were sectioned parallel to the surface of the intestine (tangential sections), parallel to their long axes in a plane through the gut wall (longitudinal sections), or at right angles to their long axes (transverse sections). In order to cut transverse or longitudinal sections, ganglia were re-embedded in TAAB resin in flat molds after the initial polymerization. Silver to pale gold ultrathin sections were cut with a diamond knife on a Reichert UMO2 ultramicrotome, mounted on single slot grids or mesh grids, stained with Reynold's lead citrate, and examined with a Jeol 1200EX electron microscope.

Quantitation. All quantitation was done on ultrathin sections mounted on butvar-coated single slot grids. To determine the proportion of varicosities that contained SP in myenteric ganglia, ganglia were sectioned transversely so that most fibers in the neuropil were cut in cross section. Immunoreactive vesicle-containing nerve fiber profiles and nonimmunoreactive vesicle-containing nerve fiber profiles were count- ed in single transverse sections through each of 3 myenteric ganglia, which had been DAB-reacted without imidazole, from the distal ileum of each of 3 animals. Profiles were classed as vesiculated if they contained 5 or morc vesicles. The number of immunoreactive profiles that formed synapses was noted.

To determine the proportion of SP-immunoreactive varicosities that formed ultrastructurally identifiable synapses and the proportion of total synapses that were SP-immunoreactive, tangential ultrathin sections were used in order to maximize the area examined and therefore the number of synapses encountered. Two sections, which were separated by at least $4 \mu \mathrm{m}$ to prevent double counting of varicosities or synapses, were examined from one myenteric ganglion from distal small intestine from each of 3 animals. For each section, the following data were collected: the total number of SP-immunoreactive vesiculated profiles, the number of SP-immunoreactive vesiculated profiles that made synaptic contacts, and the number of nonimmunoreactive vesiculated profiles that formed synapses. Synapses were identified by the presence of fuzzy clcctron-dense material on the inner leaflet of the postsynaptic membrane and by clustering of vesicles at the presynaptic membrane.

\section{Results}

Whole-mount preparations of myenteric ganglia that had been prepared from distal or proximal small intestine and labeled with the electron microscopic immunocytochemical technique used here showed dense arrays of SP-positive nerve fibers at the light microscopic level (Fig. 1a). The distribution of SP-immunoreactive fibers in the myenteric ganglia, in the internodal strands connecting the myenteric ganglia and in the meshwork of the tertiary myenteric plexus was similar to that reported previously (e.g., Costa et al., 1980). In $1 \mu \mathrm{m}$ sections through ganglia labeled for electron microscopy (Fig. $1 b$ ), darkly stained SP-containing nerve fibers occurred around all the myenteric nerve cell bodies, forming a dense network in the neuropil of the ganglia. In antibody-labeled ganglia that had been reacted with DAB in the presence of imidazole (Fig. $1 b$ ), many of the cell bodies in the ganglia appeared to be SP-immunoreactive. The intensity of the staining of the cell bodies varied. A few cell bodies were moderately to intensely labeled, whereas the majority of the positive cells were only faintly stained. Some large and some small nerve cell bodies were immunoreactive. The small SP-positive cell bodies were often located on the surfaces of the ganglia or near or among the nerve fibers of the internodal strands where they traveled through the ganglia. When ganglia were reacted without imidazole (not shown), almost all of the cells appeared to be SP-negative, with only a few cells showing moderate to intense staining for SP.

Because SP-immunoreactive nerve fibers appeared to be densely and homogeneously distributed in myenteric ganglia at the light microscopic level, there was no basis for selecting one area of an intact SP-labeled myenteric ganglion over another for ultrastructural study. Consequently, random ultrathin sec-

\footnotetext{
Figure 1. Distribution of SP-immunoreactive nerve fibers in myenteric ganglia of guinea pig small intestine. $a$, Light micrograph of a wholemount preparation of a myenteric ganglion that was stained for the electron microscopic demonstration of SP. The ganglion contains a very dense network of varicose, SP-positive fibers. The internodal strands $(I S)$ that connect the ganglia contain varicose SP fibers. No imidazole intensification. Scale bar, $50 \mu \mathrm{m} . b$, Light micrograph of a semithin $(1 \mu \mathrm{m})$ section through a myenteric ganglion that was stained for the electron microscopic demonstration of SP. SP-positive nerve fibers containing intense peroxidase reaction product surround all the neuronal (*) cell bodies, as well as areas presumed to be the cell bodies of enteric glia (A). One of the enteric neurons (arrow) is strongly immunoreactive for SP. Imidazole intensification. $E$, extraganglionic space. Scale bar, $20 \mu \mathrm{m}$. $c$, Electron micrograph of a longitudinal section through a myenteric ganglion stained for SP. Many SPimmunoreactive nerve fiber profiles, which contain electron-dense peroxidase reaction product, occur throughout the neuropil of the ganglion. Each of the 3 nerve cell bodies $(N C B)$ in the micrograph is closely approached by several vesiculated SP-positive nerve profiles. Vesiculated SP profiles also lie on the surface of the ganglion without a covering of glial cell cytoplasm (arrowheads). No imidazole intensification. $C M$, circular muscle; $G$, enteric glial cell; $L M$, longitudinal muscle. Scale bar, $5 \mu \mathrm{m}$. $d$, Electron micrograph of a transverse section through the neuropil of a myenteric ganglion. Both vesiculated (arrow) and nonvesiculated (arrowheads) nerve fiber profiles are SP-immunoreactive. No imidazole intensification. $N C B$, nerve cell body. Scale bar, $1 \mu \mathrm{m}$.
} 
tions were used to study the ultrastructural distribution and synaptic relationships of SP-immunoreactive fibers in the ganglia.

In single ultrathin sections through myenteric ganglia from proximal and distal small intestine, many SP-positive nerve fiber profiles occurred in the neuropil (Figs. 1c,2). DAB reaction product was seen in both vesiculated and nonvesiculated nerve fiber profiles (Fig. 1d). The immunoreactive vesiculated profiles contained variable proportions of large granular vesicles (LGV) and small round vesicles. Although these small round vesicles sometimes contained peroxidase reaction product, they are probably equivalent to the small clear vesicles (SCV) seen after conventional processing. Many of the SP-positive vesiculated profiles contained mainly SCV with only occasional LGV (Figs. $2 ; 3 a-c)$, whereas a few profiles contained many LGV in addition to SCV (Fig. $3 d$ ). In ganglia that had been reacted with imidazole, some of the profiles were so intensely labeled that it was difficult to see their limiting membranes clearly. However, in ganglia that were reacted without imidazole, both SP-positive and SP-negative profiles were clearly defined. In single transverse sections through 9 ganglia from distal small intestine of 3 animals, 1665 out of 2517 (or 66\%) of the vesicle-containing profiles were found to be SP-immunoreactive (range for individual ganglia, 50.3-71.2\%; median value, $69.5 \% ; 95 \%$ confidence limit of range estimated from binomial probability distribution, $43-77 \%$ ).

In every ultrathin section examined from both distal and proximal small intestine, at least one vesicle-containing SPimmunoreactive nerve fiber profile was directly apposed to every myenteric nerve cell body (Figs. $1 c, 2$ ), i.e., the plasma membranes of the immunorcactive profiles and the cell bodies were separated by $15-20 \mathrm{~nm}$ with no intervening neuronal or glial process. In the neuropil, immunoreactive vesicle-containing profiles were also directly apposed to other immunoreactive vesiculated and nonvesiculated nerve fiber profiles and to nonimmunoreactive vesiculated and nonvesiculated profiles. Only a very small proportion of the total number of SP-immunoreactive nerve fiber profiles formed morphologically identifiable synapses with clustering of vesicles presynaptically and fuzzy electron-dense material postsynaptically. Out of a total of 21,821 SP-immunoreactive vesicle-containing profiles in 6 tangential sections through 3 myenteric ganglia from distal small intestine of 3 animals, 128 (or $0.59 \%$ ) made synaptic contacts (range for 6 individual sections, $0.40-0.69 \%$ ). Of these synapses, 57 were axosomatic (Fig. 2), and the remainder (71) were formed on nonvesiculated nerve processes of variable size (Fig. 3). No synapses by SP-positive profiles on vesicle-containing nerve fiber profiles were encountered. SP-containing synapses were also found in the sample of transverse sections through 9 ganglia from the distal small intestine of 3 animals. Out of 1665 immunoreactive profiles, 11 (or $0.66 \%$ ) were found to form synapses. Single sections through 7 ganglia contained $1-3$ synapses; single sections through 2 ganglia contained no synapses. Although very few of the SP-immunoreactive varicosities made synapses, SP synapses made up a large proportion of the total number of synapses in the myenteric ganglia. In the sample of 6 tangential sections, 143 nonimmunoreactive synapses were found in addition to the 128 immunoreactive ones. Thus, $47 \%$ of the total synapses were made by nerve fiber profiles that contained SP (range of percentages of positive synapses in each of 6 tangential sections, 43.1-55.5\%). Nonimmunoreactive synapses were also formed almost exclusively on nerve cell bodies or on nonvesiculated nerve processes. Of the 143 nonimmunoreactive synapses, only 2 occurred on vesicle-containing nerve fiber profiles. Thus, out of the sample of immunoreactive plus nonimmunoreactive synapses examined in this study, 2 out of 271 (or $0.7 \%$ ) of the synapses were made on vesiculated nerve fiber profiles. These results serve as quantitative confirmation of previous observations (e.g., Gabella, 1972; Cook and Burnstock, 1976) that synapses on vesicle-containing nerve fiber profiles are rare in myenteric ganglia of guinea pig ileum. SP-immunoreactive synapses were also found, but not quantified, in the one sample of proximal small intestine and in the one sample of extrinsically denervated small intestine that were examined.

There appeared to be a marked spatial segregation of SPimmunoreactive synapses towards the periphery of myenteric ganglia. Processes that received SP-immunoreactive synapses were usually located peripherally rather than centrally within the ganglia. Axosomatic SP synapses were preferentially formed on small neurons, the cell bodies of which lay near the surfaces of the ganglia or near the nerve fibers of the internodal strands where they traveled through the ganglia. Many of the neurons with SP synapses had processes that reached the surfaces of the ganglia and were directly exposed to the extraganglionic space without a covering of glial cell cytoplasm (Fig. 2, $d, e$ ). In ganglia that were DAB-reacted in the presence of imidazole, some of the nerve cell bodies that received SP synapses also contained SP-immunoreactivity. In one ultrathin section, a superficially located nerve cell body was found to receive 3 SP synapses; this observation suggests that around some myenteric neurons, synapse-forming SP-positive nerve fibers occur in baskets, which are concealed at the light microscopic level by the mass of SP varicosities in the neuropil.

SP-immunoreactive nerve fiber profiles in the internodal strands were also examined. Immunoreactive as well as nonimmunoreactive vesicle-containing nerve fiber profiles were concentrated near the surfaces of the internodal strands. Both positive and negative vesiculated profiles were often exposed to the extraganglionic space without a covering of glial cell cytoplasm. On the other hand, immunorcactive and nonimmunoreactive profiles without vesicles were found both centrally and peripherally within the internodal strands.

In control experiments, no labeled fibers were found by light or electron microscopy when myenteric ganglia were incubated without primary antiserum or incubated with anti-SP antiserum that had been absorbed with $10^{-5} \mathrm{M}$ SP. After being stained with anti-SP that had been absorbed with $10^{6} \mathrm{M}$ SP, myenteric ganglia contained rare immunoreactive fibers by both light and electron microscopy.

\section{Discussion}

\section{Nature of the SP-like antigen in guinea pig small intestine}

High-pressure liquid chromatography and radioimmunoassay have shown that the SP-like immunoreactivity in extracts of myenteric plexus from guinea pig ileum is due to the authentic undecapeptide (Murphy et al., 1982). Moreover, SP-like material extracted from the whole gut wall (Holzer et al., 1981) and SP-like immunoreactivity released by elevated $\mathrm{K}^{+}$concentration or electrical field stimulation (Holzer, 1984) have identical elution patterns to authentic SP on gel chromatography. Material with SP-like activity from extracts of myenteric plexus and the SP-like substance released from enteric nerves by stimulation have also been shown to act on the same receptors as authentic SP when bioassayed on guinea pig ileum (Franco et 

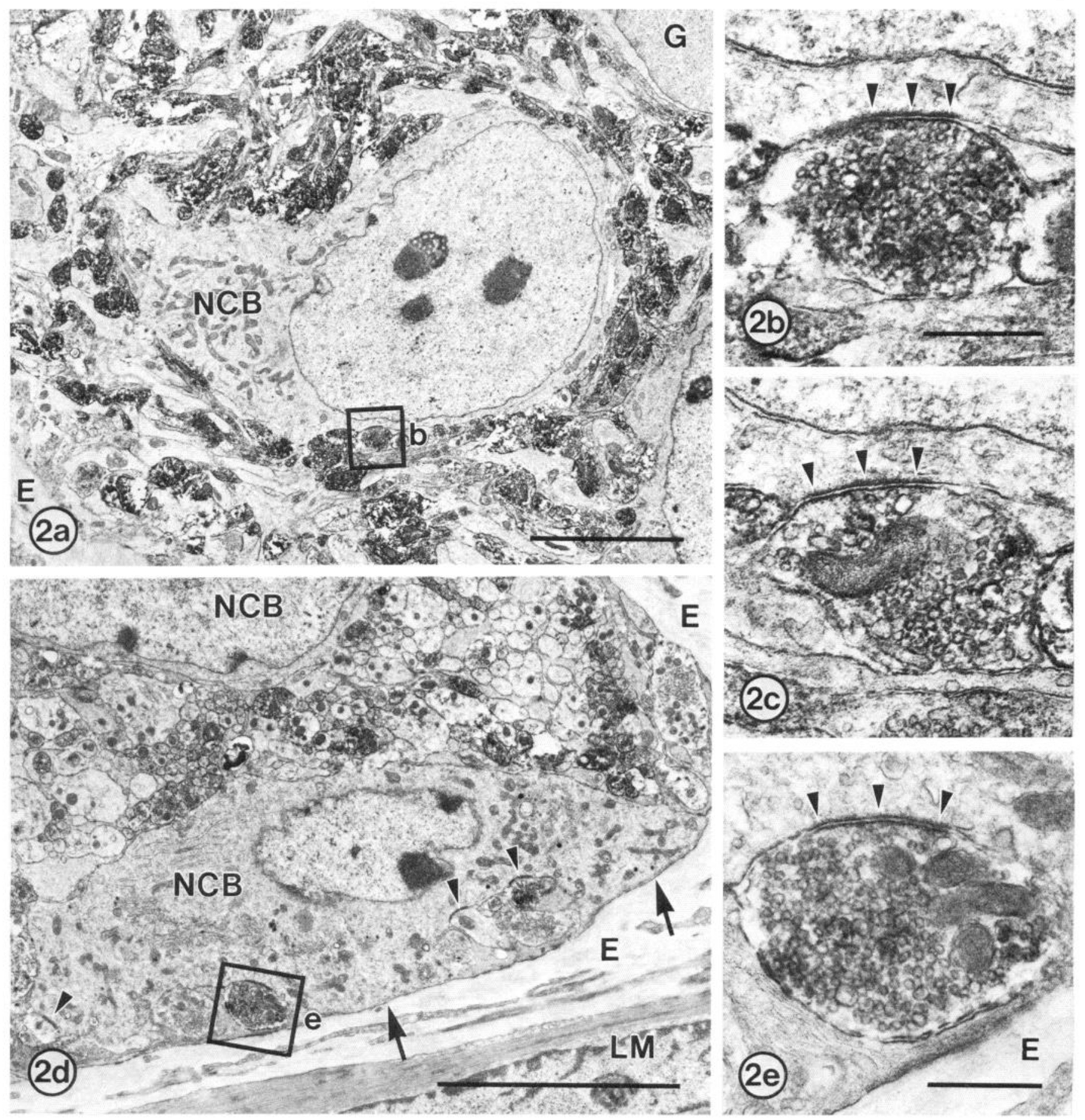

Figure 2. Electron micrographs of SP-immunoreactive synapses on nerve cell bodies in myenteric ganglia of guinea pig small intestine. $a$, The cell body $(N C B)$ of a small myenteric neuron is surrounded by many SP-positive vesicle-containing profiles. One of these profiles, which is indicated by the box marked $b$ and shown at higher magnification in $b$, forms a synapse on the cell body. There is also a dense network of SP-immunoreactive nerve fiber profiles in the neuropil. The nerve cell body lies close to the surface of the ganglion. Tangential section. No imidazole intensification. $E$, extraganglionic space; $G$, enteric glial cell. Scale bar, $5 \mu \mathrm{m}$. $b$ and $c$, Two sections through the nerve fiber profile indicated by box $b$ in $a$. The sections were separated by about $100 \mathrm{~nm}$. The synapse shows a well-defined specialization (arrowheads) on the postsynaptic membrane. Scale bar in $b, 500 \mathrm{~nm}$, applies to $b$ and $c$. $d$, A myenteric neuron receives a synapse from an immunoreactive profile, which is indicated by the box marked $e$ and shown at a higher power in $e$. Other immunoreactive and nonimmunoreactive nerve fiber profiles (arrowheads) also form synapses on the neuron. Parts of the nerve cell body $(N C B)$ reach the surface of the ganglion (arrows), where they are exposed to the extraganglionic space $(e)$ without a covering of glial cytoplasm. Transverse section. No imidazole intensification. $L M$, longitudinal muscle. Scale bar, $5 \mu \mathrm{m}$. $e$, The synapse made by the nerve fiber profile indicated by box $e$ in $d$ has an obvious postsynaptic density (arrowheads). $e$, extraganglionic space. Scale bar, 500 nm. 

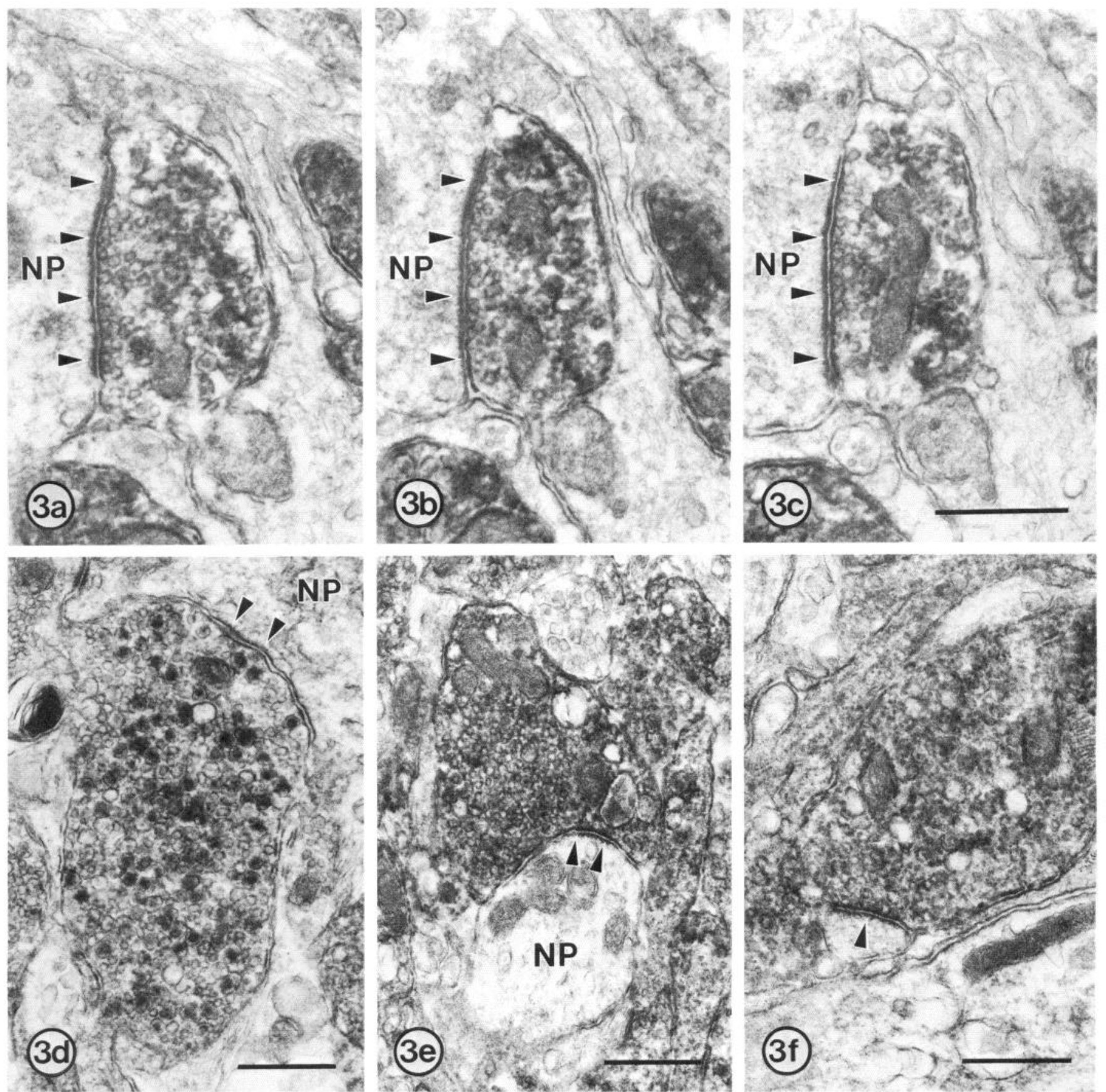

Figure 3. Electron micrographs of SP-immunoreactive synapses on nerve processes in guinea pig myenteric ganglia. $a-c$, Serial sections through SP-positive nerve fiber profile that makes a synapse on a nonvesiculated nerve process $(N P)$. The synaptic site shows a well-defined postsynaptic membrane specialization (arrowheads), and there are small round vesicles lined up along the presynaptic membrane. The positive profile contains mainly small round vesicles and a few large granular vesicles. The nerve process receiving the synapse lay within $1 \mu \mathrm{m}$ of the surface of the ganglion. Tangential section. No imidaziole intensification. Scale bar in $c, 500 \mathrm{~nm}$, applies to $a-c$. $d$, SP-containing nerve fiber profile forms a synapse (arrowheads) on a large nonvesiculated nerve process $(N P)$. The positive profile contains a high proportion of large granular vesicles. Tangential section. Imidazole intensification. Scale bar, $500 \mathrm{~nm}$. $e$, SP-immunoreactive nerve fiber profile makes a synapse (arrowheads) on a small nonvesiculated nerve process $(N P)$. Tangential section. No imidazole intensification. Scale bar, $500 \mathrm{~nm}$. $f$, A very small nonvesiculated nerve process, which is possibly a dendritic spine, receives a synapse (arrowheads) from a nerve fiber profile that contains SP immunoreactivity. Tangential section. Imidazole intensification. Scale bar, $500 \mathrm{~nm}$.

al., 1979). Furthermore, the antiserum used here does not crossreact significantly with other tachykinins that could be present in enteric nerves-neurokinin A, kassinin, physaelamin, and eledoisin (Morris et al., 1986) - and we have shown in this study that antibody labeling of intestinal nerves fixed for electron microscopy can be prevented by pre-equilibration of the antiserum with SP. All these data strongly suggest that SP in the tissue is localized by this antiserum and that the vesiculated 
profiles labeled here contain authentic SP. However, it cannot be excluded that other immunochemically similar molecules have also been detected.

\section{Vesicles in SP-immunoreactive nerve fibers}

The SP-immunoreactive profiles seen in this study contained SCV and LGV in ratios that differed markedly between individual profiles. In general, the range of variability in the proportions of SCV and LGV in SP profiles was similar to that found in profiles containing SCV and LGV from conventionally processed gut. Thus, the SP profiles have no distinctive ultrastructural feature that allows them to be differentiated from other SCV/LGV profiies. This conclusion is not surprising because all neuropeptide-immunoreactive nerve fibers in the gut studied to date contain similar types of vesicles and because neuropeptides are often colocalized in enteric neurons (see Costa et al., 1986a; Llewellyn-Smith, 1987). It also reinforces the view that immunocytochemistry is the only reliable method of distinguishing, at the ultrastructural level, nerve fibers that contain different neuropeptides.

\section{Synaptic relationships of SP-immunoreactive nerve fibers}

Synapses can be defined in 2 ways: functionally by their ability to transmit information from one neuron to another neuron or effector cell or morphologically by the structural specializations they show when viewed with an electron microscope. In the present study, we found that many vesicle-containing SP-positive profiles were directly apposed to every myenteric neuron and that in the neuropil reactive vesiculated profiles often contacted other vesiculated and nonvesiculated nerve profiles. However, only a very small proportion (about $0.6 \%$ ) of the contacts between positive profiles and other neuronal structures showed morphologically identifiable synaptic specializations with clustering of vesicle presynaptically and fuzzy electron-dense material postsynaptically. We have assumed that these ultrastructurally specialized contacts by SP profiles represent functional synapses. However, it is not clear whether the close appositions without specializations are also effective sites of neurotransmission.

Morphologically identifiable SP synapses were found to be most common on the somata of small nerve cells, which lay at the edges of the ganglia or near the internodal strands. These neurons are almost certainly $S$ neurons, one of the two electrophysiologically defined types of myentric neurons (Hirst et al., 1975) since neurons of the other electrophysiological type (AH neurons) have large cell bodies and are centrally placed within the ganglia (see below). Included among the $\mathrm{S}$ neurons are the motor neurons to the muscle (Furness et al., 1988b). Other $\mathrm{S}$ neurons are possibly interneurons in the myenteric plexus, although there is as yet no direct evidence for this assumption. Some of the small neurons that received many SP-positive synapses were themselves SP immunoreactive. SP-containing nerve fibers represent about half of the nerve fibers in the circular muscle, where they are thought to play an excitatory role (Llewellyn-Smith et al., 1988b). All the nerve fibers to the circular muscle arise from nerve cell bodies in the myenteric plexus (Wilson et al., 1987). These data are consistent with the suggestion that at least some of the small myenteric neurons that receive ultrastructurally identifiable SP-immunoreactive synapses are motor neurons to the muscle. What roles these SPimmunoreactive inputs might play in the microcircuitry of the enteric nervous system is not known, but they are very likely to be excitatory (Katayama and North, 1978; Katayama et al., 1979).

$\mathrm{AH}$ neurons constitute the other electrophysiologically defined type of myenteric neuron. Between 10 and $35 \%$ of myenteric neurons belong to this group (Furness and Costa, 1987). These neurons have large, smooth cell bodies (Dogiel Type II morphology), are usually located centrally within the ganglia, and often give rise to several long processes (Bornstein et al., 1984a; Erde et al., 1985; Iyer et al., 1988). About $85 \%$ of $\mathrm{AH}$ neurons are immunoreactive for calcium binding protein (calbindin $\mathrm{D} 28 \mathrm{~K}$ ) and do not provide terminals to the muscle (Furness et al., 1988a; Iyer et al., 1988). The other AH neurons are also presumed not to supply the muscle. AH neurons receive morphologically identifiable synapses (Erde et al., 1985; Llewellyn-Smith et al., 1988a; Pompolo and Furness, 1988), but this study indicates that few, if any, of these are likely to be SPimmunoreactive. However, each $\mathrm{AH}$ cell undoubtedly receives a large number of ultrastructurally unspecialized contacts from SP-positive vesiculated nerve fiber profiles, since this is the case for all myenteric neurons. On the basis of electrophysiological experiments, SP has been suggested to contribute to slow esps in both S and AH neurons (Katayama and North, 1978; Katayama et al., 1979; Morita et al., 1980; Johnson et al., 1981; Bornstein et al., 1984b). The results of this study suggest that if SP is involved in generating slow esps in $\mathrm{AH}$ cells, it is likely to act through morphologically nonspecialized contacts rather than through contacts that show the generally accepted ultrastructural features of a synapse.

\section{References}

Baron, S. A., B. M. Jaffe, and A. R. Gintzler (1983) Release of substance $P$ from the enteric nervous system: Direct quantitation and characterization. J. Pharmacol. Exp. Ther. 227: 365-368.

Bartho, L., and P. Holzer (1985) Search for a physiological role of substance $P$ in gastrointestinal motility. Neuroscience 16:1-32.

Bartho, L., P. Holzer, J. Donnerer, and F. Lembeck (1982) Evidence for the involvement of substance $P$ in the atropine-resistant peristalsis of the guinea-pig ileum. Neurosci. Lett. 32: 69-74.

Bornstein, J. C., M. Costa, J. B. Furness, and G. M. Lees (1984a) Electrophysiology and enkephalin immunoreactivity of identified myenteric plexus neurones of guinea-pig small intestine. J. Physiol. (Lond.) 351: 313-325.

Bornstein, J. C., R. A. North, M. Costa, and J. B. Furness (1984b) Excitatory synaptic potentials due to activation of neurons with short projections in the myenteric plexus. Neuroscience 11: 723-732.

Cook, R. D., and G. Burnstock (1976) The ultrastructure of Auerbach's plexus. I. Neuronal elements. J. Neurocytol. 5: 171-194.

Costa, M., A. C. Cuello, J. B. Furness, and R. Franco (1980) Distribution of enteric neurons showing immunoreactivity for substance $P$ in the guinea-pig ileum. Neuroscience 5: 323-331.

Costa, M., J. B. Furness, and I. J. Llewellyn-Smith (1986a) Histochemistry of the enteric nervous system. In Physiology of the Gastrointestinal Tract, L. R. Johnson, ed., pp. 1-40, Raven, New York

Costa, M., J. B. Furness, I. J. Llewellyn-Smith, R. Murphy, J. C. Bornstein, and J. R. Keast (1986b) Functional roles for substance P-containing neurones in the gastrointestinal tract. In Substance P. Metabolism and Biological Actions, C. C. Jordan and P. Oehme, eds., pp. 99-1 19, Taylor and Francis, London.

Erde, S. M., D. Sherman, and M. D. Gershon (1985) Morphology and serotonergic innervation of physiologically identified cells of the guinea pig's myenteric plexus. J. Neurosci. 5: 617-633.

Fehér, E., and T. Wenger (1981) Ultrastructural immunocytochemical localization of substance P in the cat small intestine. Acta Anz. Jena 150: 137-143.

Fehér, E., Cs. Léránth, and T. H. Williams (1984) Ultrastructural identification of substance $P$ cells and processes in the rat myenteric plexus. Acta Morphol. Hung. 32: 23-29.

Franco, R., M. Costa, and J. B. Furness (1979) Evidence for the release 
of endogenous substance $P$ from intestinal nerves. Naunyn Schmiedeberg's Arch. Pharmacol. 306: 195-201.

Furness, J. B., and M. Costa (1987) The Enteric Nervous System, Churchill-Livingstone, Edinburgh.

Furness, J. B., J. R. Keast, S. Pompolo, J. C. Bornstein, M. Costa, P. C. Emson, and D. E. M. Lawson (1988a) Immunohistochemical evidence for the presence of calcium binding proteins in enteric neurons. Ccll Tissuc Res. 252: 79-87.

Furness, J. B., I. J. Llewellyn-Smith, J. C. Bornstein, and M. Costa (1988b) Chemical neuroanatomy and the analysis of neuronal circuitry in the enteric nervous system. In Handbook of Chemical Neuroanatomy, C. Owman, A. Bjorklund, and T. Hökfelt, eds., Elsevier, Amsterdam (in press).

Gabella, G. (1972) Fine structure of the myenteric plexus in the guineapig ileum. J. Anat. 111: 69-97.

Grafe, P., C. J. Mayer, and J. D. Wood (1979) Evidence that substance $\mathrm{P}$ does not mediate slow synaptic excitation within the myenteric plexus. Nature 279: 720-721.

Hedqvist, $P_{\text {., }}$ and U. S. von Euler (1975) Influence of substance $\mathbf{P}$ on the response of guinea-pig ileum to transmural nerve stimulation. Acta Physiol. Scand. 95: 341-343.

Hirst, G. D. S., M. E. Holman, and I. Spence (1975) Two types of neurones in the myentric plexus of duodenum in the guinea-pig. $\mathbf{J}$. Physiol. (Lond.) 236: 303-326.

Holzer, P. (1984) Characterization of the stimulus-induced release of immunoreactive substance $P$ from the myenteric plexus of the guineapig small intestine. Brain Res. 297: 127-136.

Holzer, P., and F. Lembeck (1979) Effect of neuropeptides on the efficiency of the peristaltic reflex. Naunyn Schmiedeberg's Arch. Pharmacol. 307: 257-264.

Holzer, P., and F. Lembeck (1980) Neurally mediated contraction of ileal longitudinal muscle by substance P. Neurosci. Lett. 17: 101105 .

Holzer, P., P. C. Emson, L. L. Iversen, and D. F. Sharman (1981) Regional differences in the response to substance $P$ of the longitudinal muscle and the concentration of substance $P$ in the digestive tract of the guinea-pig. Neuroscience 6: 1433-1441.

Iyer, V., J. C. Bornstein, M. Costa, J. B. Furness, Y. Takahashi, and T. Iwanaga (1988) Electrophysiology of myenteric neurons immunoreactive for a calcium binding protein in the guinea-pig. J. Autonom. Nerv. Syst. 22: 141-150.

Johnson, S. M., Y. Katayama, K. Morita, and R. A. North (1981) Mediators of slow synaptic potentials in the myenteric plexus of the guinea-pig ileum. J. Physiol. (Lond.) 320: 175-186.

Katayama, Y., and R. A. North (1978) Does substance P mediate slow synaptic excitation within the myenteric plexus? Nature 274: $387-388$.

Katayama, Y., R. A. North, and J. T. Williams (1979) The action of substance $\mathrm{P}$ on neurons of the myenteric plexus of the guinea-pig small intestine. Proc. R. Soc. London 206: 191-208.
Leander, S., R. Håkanson, and F. Sundler (1981) Nerves containing substance $P$, vasoactive intestinal polypeptide, enkephalin or somatostatin in the guinea-pig taenia coli. Distribution, ultrastructure and possible functions. Cell Tissue Res. 215: 21-39.

Llewellyn-Smith, I. J. (1987) Neuropeptides and the microcircuitry of the enteric nervous system. Experientia 43: 813-821.

Llewellyn-Smith, I. J., J. B. Furness, R. Murphy, P. E. O'Brien, and M. Costa (1984) Substance P-containing nerves in the human small intestine. Distribution, ultrastructure and characterization of the immunoreactive peptide. Gastroenterology 86: $421-435$.

Llewellyn-Smith, I. J., M. Costa, and J. B. Furness (1985) Light and electron microscopic immunocytochemistry of the same nerves from whole mount preparations. J. Histochem. Cytochem. 33: 857-866.

Llewellyn-Smith, I. J., J. C. Bornstein, and H. Fujisawa (1988a) Synapses on electrophysiologically-characterized myenteric neurons identified with antibodies to Lucifer Yellow. Neurosci. Lett. Suppl. 30: $\mathbf{S 9 3 .}$

Llewellyn-Smith, I. J., J. B. Furness, I. L. Gibbins, and M. Costa (1988b) Quantitative ultrastructural analysis of enkephalin-, substance P- and VIP-immunoreactive nerve fibers in the circular muscle of the guineapig small intestine. J. Comp. Neurol. 272: 139-148.

Morita, K., R. A. North, and Y. Katayama (1980) Evidence that substance $\mathrm{P}$ is a neurotransmitter in the myenteric plexus. Nature 287: $151-152$.

Morris, J. L., I. L. Gibbins, G. Campbell, R. Murphy, J. B. Furness, and M. Costa (1986) Innervation of the large arteries and heart of the toad (Bufo marinus) by adrenergic and peptide-containing neurons. Cell Tissue Res. 243: 171-184.

Murphy, R., J. B. Furness, A. M. Beardsley, and M. Costa (1982) Characterization of substance P-like immunoreactivity in peripheral sensory nerves and enteric nerves by high pressure liquid chromatography and radioimmunoassay. Regulat. Peptides 4: 203-212.

Pernow, B. (1983) Substance P. Pharmacol. Rev. 35: 85-141.

Pompolo, S., and J. B. Furness (1988) Ultrastructure of Dogiel Type II cells revealed by immunoreactivity for calcium binding protein $(\mathrm{CaBP})$ in the myenteric plexus of guinea-pig ileum. Neurosci. Lett. Suppl. 30: S111.

Straus, W. (1982) Imidazole increases the sensitivity of the cytochemical reaction for peroxidase with diaminobenzidine at a neutral $\mathrm{pH}$. J. Histochem. Cytochem. 30: 491-493.

Tonini, M., G. Frigo, S. Lecchini, L. D'Angelo, and A. Crema (1981) Hyoscine-resistant peristalsis in guinea-pig ileum. Eur. J. Pharmacol. 71: 375-381.

von Euler, U. S., and J. H. Gaddum (1931) An unidentified depressor substance in certain tissue extracts. J. Physiol. (Lond.) 72: 74-78.

Wilson, A. J., I. J. Llewellyn-Smith, J. B. Furness, and M. Costa (1987) The source of the nerve fibres forming the deep muscular and circular muscle plexuses in the small intestine of the guinea-pig. Cell Tissue Res. 247: 497-504. 